\title{
Seismic Analysis of Structures By Etabs in B.S Code \& I.S Code
}

\author{
Muhaned Abass Mohammed \\ Jawaharlal Nehru Technological University \\ Anantapuramu
}

\begin{abstract}
Earth quick has become popular in $20^{\text {th }}$ century in whole world. It's relating with safety so we must be care about designing and analysis of Earth quick. Since past Earth quick happen in expected countries in expected part in continent. Nature disasters had changed from time to time even climate effected by time so in modern century and development of knowledge there is no expectation where are disasters will happen just we must be ready to provide and protect humans.

When we are talking about Earth quick we must know that we are talking about human souls in two options death or live there is no other choice. As we are engineers we learned in college about foundations of engineering especially in civil engineering when we are going to design structures we must keep constant principles are safety, architecture view and finally economy. So I always remember my self by occupation moral and follow it.

Due to important of seismic analysis in whole structures I am going to talk about it in my project. I will do seismic analysis and design by ETABS with IS CODE because I am doing it in INDIA and with BS CODE because I am from Africa we follow BS CODE. So main aim of my project is comparative between IS CODE and BS CODE in Seismic analysis of structures by ETABS.
\end{abstract}

Key words: Seismic analysis, structures data, analysis, design, comparative.

\section{INTRODUCTION:}

The vibrations arising from earthquakes are characterized by randomness, as the soil vibrates in the earthquake zone in various directions, which causes a variable ground acceleration that affects the foundations of origin located in the earthquake area, which in turn affects the elements of the structural structure that vibrate and generate internal forces related to the amount of acceleration due to the earthquake as well as the mass of these elements

In general, the ground acceleration due to earthquakes can be expressed by horizontal and vertical vehicles except that the vertical vehicles are neglected due to the fact that the vertical hardness is very large and the focus is on studying the installations to resist the horizontal vehicles to acceleration only.

Seismic analysis of facilities can be divided into two main groups:

1. Methods of static "static" analysis.

2. The methods of dynamic analysis include:

A- Nonlinear analysis methods.

B- Methods of direct integration of the motion equation.

C- Methods of phasing analysis using response spectrum charts.

Dynamic analysis is used in the following cases:
1- The height of the origin exceeding $(75 \mathrm{~m})$.

2- A difference in the floor height of any floor in the building.

3- A difference in the structural sentences and in the building materials formed for each of them.

4- A big difference in the shape of the horizontal projection between the repeated floors.

5- The non-continuation of one of the structural elements (column or shear wall) throughout the entire building height.

6- A big difference in the distribution of the interior partitions between successive floors.

The results obtained in various ways may be characterized by significant differences due to the nature of the assumptions used in each of them

Therefore, choosing the appropriate method for the studied structure depends on several factors and the experience of the structural engineer.

The following table can be used to choose the appropriate method:

Type of Origin - Seismic analysis method Small facilities

- Static analysis method

Huge and complex installations

- Nonlinear analysis method

Medium size facilities

- Direct integration method

Larger and more complex installations

- Phase analysis method

Explanation of the static analysis method

This method relies on converting the seismic dynamic forces into equivalent horizontal static forces that affect the building in a horizontal direction

According to the main axes of the building.

This method is used in the case of small installations or almost regular shapes with a section that is fixed or semifixed at its entire height, and that the decline in the horizontal projection does not exceed $25 \%$ of its area on the upper floors

Calculate the equivalent horizontal forces:

The static analysis method depends on finding the horizontal force caused by the earthquake and applied in the base of origin level called the base shear force.

\section{Coefficient Z:}

It is called the seismic coefficient of the region in which the studied origin is established, bearing in mind that the seismic map approved in the Arab world includes five seismic regions: 
Modified Mercury scale Description of the region Factor Z Region number

Smaller than 1 is not prone to 0 earthquakes. 0

1 is not subject to 0.1 strong earthquakes

2 area of moderate seismic damage 0.2

3 region with major seismic damage 0.3

4 Greater than 3 , a region with damaging seismic damage

0.4

\section{Parameter K:}

This coefficient represents the inelastic (plastic) behavior of facilities when exposed to seismic loads and its value decreases as the building compliance increases and its value is taken from the following table: Characteristics of the structural sentence, laboratory $\mathrm{K}$, high water tanks and the like, carried on a group of columns not less than (4) and adequately connected horizontally in both directions. 2.5 Special establishments: chimneys, minarets, television towers, cooling towers ... 2 Installations carried out from load-bearing walls of reinforced concrete, planed or in a vacuum shape (shear walls or central core) ... 1.3 Installed buildings or buildings Of structural or mixed construction frameworks according to the following design case: A- ATires and shear walls together resist horizontal loads. B- BThe shear walls are calculated to bear the entire horizontal load. (In both cases, the flat or vacuum tire resistance must not be less than $25 \%$ of the total horizontal loads.) 0.8 .

\section{1 objectives:}

This study focuses on comparison of International standards. The chosen standards are British code (British Society of civil Engineers) and Indian code i.e. IS 1893:2002. The study also helps in understanding the main contributing factors which lead to poor performance of Structure during the earthquake, so as to achieve their adequate safe behavior under future earthquakes. The structure analysed is symmetrical, G+10, Modelling of the structure is done as per Etabs software.

\subsection{Methodology:}

The methodology worked out to achieve the mentioned objectives is as follows:

- 1. Modeling of the selected building in ETABS Software.

- 2. Retrieved time history and response spectrum of structure from the software.

- 3. Two models as per the codes i.e. Indian code, British code specification were made.

- 4. Calculated push analysis seismic forces and load combinations as per IS 1893-2002, and BS 8110-1997.

- 5. Analysed the models of the data is presented to evaluate stability.

\section{LITERATURE REVIEW:}

\section{A. A. Kale, S. A. Rasal, (2017):}

In this proposed study four different shapes of same area multistorey model is generated \& tested by the ETABS under the guideline of IS-875-Part3 \& IS1893-2002-Part1. The behavior of $15,30 \& 4$ h5 storey building has been studied. The Dynamic effects also find by Response spectrum method. All the parameters like Story displacement, Story drift, Base shear, Overturning moments, Acceleration and Time period are calculated. After comparing all building shapes results concluded that which section is convenient \& either seismic or wind effect is critical.

\section{Gauri G. Kakpure, Ashok R. Mundhada (2016):}

This paper presents a review of the previous work done on multistoried buildings vis-à-vis earthquake analysis. It focuses on static and dynamic analysis of buildings. This paper presents a review of the comparison of static and dynamic analysis multistoried building. Design parameters such as Displacement, Bending moment, Base shear, Storey drift, Torsion, Axial Force were the focus of the study.

\section{G. Guruprasad. (2017):}

performed a dynamic analysis of G+15 storied RC frame building withL, C \& rectangular shapein plan with the help of ETABS software. Comparison has been done by considering the parameters such as story drift, story shear, support reactions, building mode, and section cut force. It has been concluded that maximum value of story shear was observed for L-shape plan than rectangular building and Cshape building. The stories drift values in $\mathrm{X}$ direction and $\mathrm{Y}$ direction increases for top to bottom story in all three cases. When earthquake load is applied in Y direction, it was found that irregular plan structure can resist more base shear than rectangular plan structure. Regular building and $\mathrm{L}$-shape buildings are gave good results than $\mathrm{C}$-shaped buildings in all aspect.

Athulya Ullas (2017):

performed wind analysis of buildings having various shapes such as Y, Plus and V. Buildings of plan shapes Y, Plus and V are modeled in ETABS 2016 and analyzed. It is observed that the storey force is same for all the buildings, i.e. the storey force does not change with the shape. The lateral displacement is found maximum for $\mathrm{V}$ shape building. The storey drift is observed maximum for $\mathrm{Y}$ shape as compared to that of other shapes and the lateral displacement and the storey drift are observed minimum for Plus shape building as compared to $\mathrm{Y}$ and $\mathrm{V}$ shape buildings and hence it is the most structurally stable shape among the selected shapes.

\section{Pradeep Pujar (2017):}

analysed G+9 storied irregular buildings to find their seismic performance with $\&$ without shear walls. Shapes of building plan considered for the study were I, L \& C. Three models of bare frame \&three models with shear walls were considered for the study. The models has been analysed by Equivalent static technique with the assistance of E-tabs V 15.0.0 programming. The comparison has been done by considering the parameters such as story displacement, story drift and base shear. It has been concluded that Lshape, C-shape structures with Shear walls are having great outcomes in base shear, story drift and displacement. In all shapes the I-shape building with shear wall is having increased base shear both in $\mathrm{X}$ and $\mathrm{Y}$ direction and the $\mathrm{L}$ shape is having very less increased base shear. The building with shear wall gives better execution against the seismic tremor when compared with bare frame building.

Aniket A. Kale (2017): 
carried out the wind \& seismic analysis $15,30 \& 45$ storied buildings of four different shapes of same area by using advance software CSI ETABS. Response spectrum method was used to find the dynamic effects. The comparison has been done by considering the parameters such as story displacement, story drift, base shear, overturning moments $\mathrm{Mz}$, acceleration \& time period. It has been concluded that for maximum earthquake structure of 15-storey is most stable structure \&for maximum wind effect triangular structure of 15-storey is most stable. For 45 -storey circular \& rectangular shape building is most stable for maximum earthquake \& wind effect respectively. Wind effect is critical for 45 storey building \& on the other hand seismic is critical at 15 storey \& 30 storey building. Wind effect is more critical than earthquake.

\section{Pardeshi Sameer (2016):}

In this study, 3D analytical model of $\mathrm{G}+15$ storied buildings have been generated for symmetric and asymmetric building models and analyzed using structural analysis tool ETABS software. Mass and stiffness are two basic parameters to evaluate the dynamic response of a structural system.

This paper is concerned with the effects of various vertical irregularities on the seismic response of a structure. The objective of the project is to carry out Response spectrum analysis (RSA) of regular and irregular RC building frames and Time history Analysis (THA) of regular RC building frames and carry out the ductility based design using IS 13920 corresponding to response spectrum analysis. Comparison of the results of analysis of irregular structures with regular structure is done.

\section{S.Mahesh, B.Panduranga Rao (2014):}

In this paper a residential of $\mathrm{G}+11$ multi-story building is studied for earth quake and wind load using ETABS and STAAS PRO V8i .Assuming that material property is linear static and dynamic analysis are performed. These analysis are carried out by considering different seismic zones and for each zone the behaviour is assessed by taking three different types of soils namely Hard, Medium and Soft .Different response like story drift, displacements base shear are plotted for different zones and different types of soils.

\section{S.K. Ahirwar, S.K. Jain and M. M. Pande (2008):}

This paper presents the seismic load estimation for multistorey buildings as per IS: 1893-1984 and IS: 18932002 recommendations. Four multistorey RC framed buildings ranging from three storeyed to nine storeyed are considered and analyzed. The process gives a set of five individual analysis sequences for each building and the results are used to compare the seismic response viz. storey shear and base shear computed as per the two versions of seismic code. The seismic forces, computed by IS: 18932002 are found to be significantly higher, the difference varies with structure properties. It is concluded that such study needs to be carried out for individual structure to predict seismic vulnerability of RC framed buildings that were designed using earlier code and due to revisions in the codal provisions may have rendered unsafe.

\section{Dr. Sanjay K. Kulkarni 2018):}

This paper presents the seismic load estimation for multistory buildings as per IS: 1893-2002 and IS: 18932016 recommendations. The method of analysis and design of multi-storey $(\mathrm{G}+4)$ residential building located in zone III, IV. The scope behind presenting this project is to learn relevant Indian standard codes are used for design of various building element such as beam, column, slab, foundation and stair case using a software E-tab under the seismic load and wind load acting the structure. To find out the values in project base shear, time period, maximum story displacement.

\section{Gauri G. Kakpure (2017):}

Reinforced Concrete (RC) building frames are most common types of constructions in urban India. These are subjected to several types of forces during their lifetime, such as static forces due to dead and live loads and dynamic forces due to earthquake. In the present work, two tall buildings (a $\mathrm{G}+10$ and a $\mathrm{G}+25$ structure), presumed to be situated in seismic zone III, are analyzed by using two different methods viz. equivalent static analysis method and response spectrum method, using ETAB 15 software. From analysis results, the parameters like storey drift, storey displacement, Axial Load, Bending Moments are determined for comparative study. Results established the superiority of the Response spectrum method over the Equivalent static analysis method. Storey drift value for $\mathrm{G}+10$ and $\mathrm{G}+25$ are 22 to $25 \%$ less respectively, in dynamic analysis than static analysis. All the values are within the limits as per code requirement. As the height of storey increases, the displacement values too gradually increase. Top storey has maximum displacement value in both X-Y directions. For dynamic analysis, storey displacement for $\mathrm{G}+10$ and $\mathrm{G}+25$ buildings are $22 \%$ \& $26 \%$ less than the corresponding values in static analysis.

\section{B. Gireesh Babu (2017):}

In this study the seismic response of the structures is investigated under earthquake excitation expressed in the form of member forces, joint displacement, support reaction and story drift. The response is investigated for g+7 building structures by using STAAD PRO designing software. Its observed the response reduction of cases Ordinary moment resisting frame. In this case, we have taken earthquake zone 2, response factor 3 for Ordinary moment resisting frame and importance factor 1. Initially, started with the designing of simple 2-dimensional frames and manually checked the accuracy of the software with our results. Then according to the specified criteria assigned it analyses the structure and designs the members with reinforcement details for $\mathrm{G}+7$ residential building $\mathrm{RCC}$ frames. In the earthquake resistant design of $\mathrm{G}+7 \mathrm{RC}$ framed building the steel quantity increased by $1.517 \%$ to the convention concrete design. The steel quantity increased in the structure ground floor to higher floor i.e G+7 level of the structure The Storey drift condition for considered $\mathrm{G}+7$ building, the base drift $=0.0$ at every story. This says that the structure is safe under drift condition. Hence shear walls, braced columns are not necessary to be provided. Hence story drift condition is checked for the G+7 building. 


\subsection{ANALYSIS BY B.S CODE:}

\section{ANALYSIS:}

\section{Structure Data}

This chapter provides model geometry information, including items such as story levels, point coordinates, and element connectivity.

\subsection{Story Data}

Table 1.1 - Story Data

\begin{tabular}{|c|c|c|c|c|c|}
\hline Name & $\begin{array}{c}\text { Height } \\
\mathbf{m m}\end{array}$ & $\begin{array}{c}\text { Elevation } \\
\mathbf{M m}\end{array}$ & $\begin{array}{c}\text { Master } \\
\text { Story }\end{array}$ & \multicolumn{2}{|c|}{ Similar To } \\
\hline Story10 & 3000 & 30500 & No & None & No \\
\hline Story9 & 3000 & 27500 & Yes & None & No \\
\hline Story8 & 3000 & 24500 & No & Story9 & No \\
\hline Story7 & 3000 & 21500 & No & Story9 & No \\
\hline Story6 & 3000 & 18500 & No & Story9 & No \\
\hline Story5 & 3000 & 15500 & No & Story9 & No \\
\hline Story4 & 3000 & 12500 & No & Story9 & No \\
\hline Story3 & 3000 & 9500 & No & Story9 & No \\
\hline Story2 & 3000 & 6500 & No & Story9 & No \\
\hline Story1 & 3500 & 3500 & No & Story9 & No \\
\hline Base & 0 & 0 & No & None & No \\
\hline
\end{tabular}

\section{Loads}

This chapter provides loading information as applied to the model.

\subsection{Load Patterns}

\begin{tabular}{|c|c|c|c|}
\hline Name & Type & $\begin{array}{l}\text { Self Weight } \\
\text { Multiplier }\end{array}$ & Auto Load \\
\hline Dead & Dead & 1 & \\
\hline Live & Live & 0 & \\
\hline EX & Seismic & 0 & UBC 97 \\
\hline EY & Seismic & 0 & UBC 97 \\
\hline wind $x$ & Wind & 0 & BS 6399-95 \\
\hline windy & Wind & 0 & BS 6399-95 \\
\hline
\end{tabular}

\subsection{Functions}

2.2.1 Response Spectrum Functions

Table 2.2 - Response Spectrum Function - UBC 97

\begin{tabular}{|c|c|c|c|c|c|}
\hline Name & $\begin{array}{l}\text { Period } \\
\text { sec }\end{array}$ & Acceleration & Damping & $\mathrm{Ca}$ & $\mathrm{Cv}$ \\
\hline BS RS & 0 & 0.4 & 5 & 0.4 & 0.4 \\
\hline BS RS & 0.08 & 1 & & & \\
\hline BS RS & 0.4 & 1 & & & \\
\hline BS RS & 0.6 & 0.666667 & & & \\
\hline BS RS & 0.8 & 0.5 & & & \\
\hline BS RS & 1 & 0.4 & & & \\
\hline BS RS & 1.2 & 0.333333 & & & \\
\hline BS RS & 1.4 & 0.285714 & & & \\
\hline BS RS & 1.6 & 0.25 & & & \\
\hline BS RS & 1.8 & 0.222222 & & & \\
\hline BS RS & 2 & 0.2 & & & \\
\hline BS RS & 2.5 & 0.16 & & & \\
\hline BS RS & 3 & 0.133333 & & & \\
\hline BS RS & 3.5 & 0.114286 & & & \\
\hline BS RS & 4 & 0.1 & & & \\
\hline BS RS & 4.5 & 0.088889 & & & \\
\hline BS RS & 5 & 0.08 & & & \\
\hline BS RS & 5.5 & 0.072727 & & & \\
\hline BS RS & 6 & 0.066667 & & & \\
\hline BS RS & 6.5 & 0.061538 & & & \\
\hline BS RS & 7 & 0.057143 & & & \\
\hline BS RS & 7.5 & 0.053333 & & & \\
\hline BS RS & 8 & 0.05 & & & \\
\hline BS RS & 8.5 & 0.047059 & & & \\
\hline BS RS & 9 & 0.044444 & & & \\
\hline BS RS & 9.5 & 0.042105 & & & \\
\hline BS RS & 10 & 0.04 & & & \\
\hline
\end{tabular}




\subsection{Load Cases}

Table 2.3 - Load Cases - Summary

\begin{tabular}{|c|c|}
\hline Name & Type \\
\hline Dead & Linear Static \\
\hline Live & Linear Static \\
\hline EX & Linear Static \\
\hline EY & Linear Static \\
\hline wind & Linear Static \\
\hline windy & Linear Static \\
\hline RS X & Response Spectrum \\
\hline RS Y & Response Spectrum \\
\hline TH X & Nonlinear Modal History (FNA) \\
\hline TH Y & Nonlinear Modal History (FNA) \\
\hline push X & Nonlinear Static \\
\hline push Y & Nonlinear Static \\
\hline
\end{tabular}

\subsection{ANALYSIS BY I.S CODE:}

\section{Structure Data}

This chapter provides model geometry information, including items such as story levels, point coordinates, and element connectivity.

\subsection{Story Data}

Table 1.1 - Story Data

\begin{tabular}{|c|c|c|c|c|c|}
\hline Name & $\begin{array}{c}\text { Height } \\
\mathbf{m m}\end{array}$ & $\begin{array}{c}\text { Elevation } \\
\mathbf{m m}\end{array}$ & $\begin{array}{c}\text { Master } \\
\text { Story }\end{array}$ & Similar To & Splice Story \\
\hline Story10 & 3000 & 30500 & No & None & No \\
\hline Story9 & 3000 & 27500 & Yes & None & No \\
\hline Story8 & 3000 & 24500 & No & Story9 & No \\
\hline Story7 & 3000 & 21500 & No & Story9 & No \\
\hline Story6 & 3000 & 18500 & No & Story9 & No \\
\hline Story5 & 3000 & 15500 & No & Story9 & No \\
\hline Story4 & 3000 & 12500 & No & Story9 & No \\
\hline Story3 & 3000 & 9500 & No & Story9 & No \\
\hline Story2 & 3000 & 6500 & No & Story9 & No \\
\hline Story1 & 3500 & 3500 & No & Story9 & No \\
\hline Base & 0 & 0 & No & None & No \\
\hline
\end{tabular}

\section{Loads}

This chapter provides loading information as applied to the model.

\subsection{Load Patterns}

\begin{tabular}{c|c|c|c|} 
Name & Type & $\begin{array}{c}\text { Self } \\
\text { Weight } \\
\text { Multiplie }\end{array}$ & $\begin{array}{c}\text { Auto } \\
\text { Load }\end{array}$ \\
\hline Dead & Dead & 1 & \\
Live & Live & 0 & IS1893 \\
EX & Seismic & 0 & 2002 \\
\hline EY & Seismic & 0 & $\begin{array}{c}\text { IS1893 } \\
2002\end{array}$ \\
\hline windx & Wind & 0 & $\begin{array}{c}\text { Indian } \\
\text { IS875:198 } \\
\end{array}$ \\
& & & 7 \\
windy & Wind & 0 & Indian \\
& & & 7 \\
\hline
\end{tabular}




\subsection{Functions}

\subsubsection{Response Spectrum Functions}

Table 2.2 - Response Spectrum Function - IS 1893:2002

\begin{tabular}{|c|c|c|c|c|c|}
\hline Name & $\begin{array}{c}\text { Period } \\
\text { sec }\end{array}$ & $\begin{array}{c}\text { Acceleratio } \\
\text { n }\end{array}$ & Damping & Z & Soil Type \\
\hline RS & 0 & 0.24 & 5 & 0.24 & II \\
\hline RS & 0.1 & 0.6 & & & \\
\hline RS & 0.55 & 0.6 & & & \\
\hline RS & 0.8 & 0.408 & & \\
\hline RS & 1 & 0.3264 & & & \\
\hline RS & 1.2 & 0.272 & & \\
\hline RS & 1.4 & 0.233143 & & \\
\hline RS & 1.6 & 0.204 & & \\
\hline RS & 1.8 & 0.181333 & & \\
\hline RS & 2 & 0.1632 & & \\
\hline RS & 2.5 & 0.13056 & & & \\
\hline RS & 3 & 0.1088 & & \\
\hline RS & 3.5 & 0.093257 & & \\
\hline RS & 4 & 0.0816 & & \\
\hline RS & 4.5 & 0.0816 & & \\
\hline RS & 5 & 0.0816 & & \\
\hline RS & 5.5 & 0.0816 & & \\
\hline RS & 6 & 0.0816 & & \\
\hline RS & 6.5 & 0.0816 & & \\
\hline RS & 7 & 0.0816 & & \\
\hline RS & 8.5 & 0.0816 & & \\
\hline RS & 8.5 & 0.0816 & & \\
\hline RS & 9 & 0.0816 & & \\
\hline RS & 9.5 & 0.0816 & & \\
\hline RS & 10 & 0.0816 & & \\
\hline & & & & \\
\hline
\end{tabular}

\subsection{Load Cases}

Table 2.3 - Load Cases - Summary

\begin{tabular}{|c|c|}
\hline Name & Type \\
\hline Dead & Linear Static \\
\hline Live & Linear Static \\
\hline EX & Linear Static \\
\hline EY & Linear Static \\
\hline windx & Linear Static \\
\hline windy & Linear Static \\
\hline RS X & Response Spectrum \\
\hline RS Y & Response Spectrum \\
\hline TH X & Nonlinear Modal \\
\hline TH Y & History (FNA) \\
\hline push X & History (FNA) \\
\hline push Y & Nonlinear Static \\
\hline
\end{tabular}

\section{DESIGN:}

4.1Pier Design by IS 456:2000:

DESIGN PX2 \&PY2\&PY3 FOR ALL STORIES:

Pier Details

\begin{tabular}{c|c|c|c|c|c|c}
\hline Story ID & Pier ID & Centroid X (mm) & Centroid Y (mm) & Length $(\mathbf{m m})$ & Thickness $(\mathbf{m m})$ & LLRF \\
\hline Story8 & Py3 & 7300 & 6250 & 1500 & 250 & 0.9 \\
\hline \multicolumn{7}{|c}{ Material Properties } \\
\cline { 2 - 6 } & \multicolumn{7}{|c|}{ Lt.Wt Factor (Unitless) } & $\mathbf{f}_{\mathbf{y}}(\mathbf{M P a})$ & $\mathbf{f}_{\mathbf{y s}}(\mathbf{M P a})$ \\
\hline
\end{tabular}


Design Code Parameters

\begin{tabular}{c|c|c|c|c|c|c}
\hline $\boldsymbol{\Gamma}_{\mathbf{S}}$ & $\boldsymbol{\Gamma}_{\mathbf{C}}$ & $\mathbf{I P}_{\mathrm{MAX}}$ & $\mathbf{I P}_{\mathrm{MIN}}$ & $\mathbf{P}_{\mathrm{MAX}}$ & MinEcc Major & MinEcc Minor \\
\hline 1.15 & 1.5 & 0.04 & 0.0025 & 0.8 & Yes & Yes \\
\hline
\end{tabular}

Pier Leg Location, Length and Thickness

\begin{tabular}{c|c|c|c|c|c|c|c}
\hline $\begin{array}{c}\text { Station } \\
\text { Location }\end{array}$ & ID & $\begin{array}{c}\text { Left } \mathbf{X}_{\mathbf{1}} \\
\mathbf{m m}\end{array}$ & $\begin{array}{c}\text { Left } \mathbf{Y}_{\mathbf{1}} \\
\mathbf{M m}\end{array}$ & $\begin{array}{c}\text { Right } \mathbf{X}_{\mathbf{2}} \\
\mathbf{m m}\end{array}$ & $\begin{array}{c}\text { Right } \mathbf{Y}_{\mathbf{2}} \\
\mathbf{m m}\end{array}$ & $\begin{array}{c}\text { Length } \\
\mathbf{m m}\end{array}$ & $\begin{array}{c}\text { Thickness } \\
\text { mm }\end{array}$ \\
\hline Top & Leg 1 & 7300 & 5500 & 7300 & 7000 & 1500 & 250 \\
\hline Bottom & Leg 1 & 7300 & 5500 & 7300 & 7000 & 1500 & 250 \\
\hline
\end{tabular}

Flexural Design for $\mathrm{P}_{\mathrm{u}}, \mathrm{M}_{\mathrm{u} 2}$ and $\mathrm{M}_{\mathrm{u} 3}$

\begin{tabular}{c|c|c|c|c|c|c|c|c}
\hline $\begin{array}{c}\text { Station } \\
\text { Location }\end{array}$ & $\begin{array}{c}\text { Required } \\
\text { Rebar Area }\left(\mathbf{m m}^{2}\right)\end{array}$ & $\begin{array}{c}\text { Required } \\
\text { Reinf Ratio }\end{array}$ & $\begin{array}{c}\text { Current } \\
\text { Reinf Ratio }\end{array}$ & $\begin{array}{c}\text { Flexural } \\
\text { Combo }\end{array}$ & $\begin{array}{c}\mathbf{P}_{\mathbf{u}} \\
\mathbf{k N}\end{array}$ & $\begin{array}{c}\mathbf{M}_{\mathbf{u} 2} \\
\mathbf{k N}-\mathbf{m}\end{array}$ & $\begin{array}{c}\mathbf{M}_{\mathbf{u}} \\
\mathbf{k N}-\mathbf{m}\end{array}$ & $\begin{array}{c}\mathbf{P i e r}_{\mathbf{g}} \\
\mathbf{m m} \mathbf{m}^{2}\end{array}$ \\
\hline Top & 938 & 0.0025 & 0.0029 & DWal32 & 460.0522 & -13.6784 & -19.129 & 375000 \\
\hline Bottom & 938 & 0.0025 & 0.0029 & DWal32 & 485.3647 & 9.7073 & -140.1207 & 375000 \\
\hline
\end{tabular}

Shear Design

\begin{tabular}{c|c|c|c|c|c|c|c|c}
\hline $\begin{array}{c}\text { Station } \\
\text { Location }\end{array}$ & ID & $\begin{array}{c}\text { Rebar } \\
\mathbf{m m}^{2} / \mathbf{m}\end{array}$ & Shear Combo & $\begin{array}{c}\mathbf{P}_{\mathbf{u}} \\
\mathbf{k N}\end{array}$ & $\begin{array}{c}\mathbf{M}_{\mathbf{u}} \\
\mathbf{k N}-\mathbf{m}\end{array}$ & $\begin{array}{c}\mathbf{V}_{\mathbf{u}} \\
\mathbf{k N}\end{array}$ & $\begin{array}{c}\mathbf{V}_{\mathbf{c}} \\
\mathbf{k N}\end{array}$ \\
\hline Top & Leg 1 & 625 & DWal20 & 690.0731 & 196.9117 & -132.7328 & 103.0097 & 337.7923 \\
\hline Bottom & Leg 1 & 625 & DWal20 & 732.2606 & -201.2867 & -132.7328 & 103.9884 & 338.7711 \\
\hline
\end{tabular}

Boundary Element Check

\begin{tabular}{c|c|c|c|c|c|c|c}
\hline $\begin{array}{c}\text { Station } \\
\text { Location }\end{array}$ & ID & $\begin{array}{c}\text { Edge } \\
\text { Length }(\mathbf{m m})\end{array}$ & $\begin{array}{c}\text { Governing } \\
\text { Combo }\end{array}$ & $\begin{array}{c}\mathbf{P}_{\mathbf{u}} \\
\mathbf{k N}\end{array}$ & $\begin{array}{c}\mathbf{M}_{\mathbf{u}} \\
\mathbf{k N}-\mathbf{m}\end{array}$ & $\begin{array}{c}\text { Stress Comp } \\
\mathbf{M P a}\end{array}$ & $\begin{array}{c}\text { Stress Limit } \\
\mathbf{M P a}\end{array}$ \\
\hline Top-Left & Leg 1 & 0 & DWal32 & 460.0522 & -19.129 & 1.43 \\
\hline Top-Right & Leg 1 & 0 & DWal32 & 690.0731 & 196.9117 & 3.94 & 6 \\
\hline Bottom-Left & Leg 1 & 0 & DWal20 & 732.2606 & -201.2867 & 6.1 \\
\hline Botttom-Right & Leg 1 & 0 & DWal20 & 485.3647 & 25.5318 & 1.57 \\
\hline
\end{tabular}

4.2Pier Design BS 8110-97 :

Design of pier PX2, PY2,PY3 FOR ALL STORIES:

\begin{tabular}{c|c|c|c|c|c|c}
\hline Story ID & Pier ID & Centroid X $(\mathbf{m m})$ & Centroid Y $(\mathbf{m m})$ & Length $(\mathbf{m m})$ & Thickness $(\mathbf{m m})$ & LLRF \\
\hline Story4 & Px2 & 6450 & 5500 & 1500 & 250 & 0.5 \\
\hline
\end{tabular}

Material Properties

\begin{tabular}{c|c|c|c|c}
\hline $\mathbf{E}_{\mathbf{c}}(\mathbf{M P a})$ & $\mathbf{f}_{\mathrm{cu}}(\mathbf{M P a})$ & Lt.Wt Factor (Unitless) & $\mathbf{f}_{\mathbf{y}}(\mathbf{M P a})$ & $\mathbf{f}_{\mathbf{y s}}(\mathbf{M P a})$ \\
\hline 31000 & 25 & 1 & 360 & 360 \\
\hline
\end{tabular}

Design Code Parameters

\begin{tabular}{c|c|c|c|c|c}
\hline Уc & Ys & УM & IP $_{\text {MAX }}$ & IP $_{\text {MIN }}$ & $\mathbf{P}_{\text {MAX }}$ \\
\hline 1.5 & 1.15 & 1.25 & 0.04 & 0.0025 & 0.8 \\
\hline
\end{tabular}

Pier Leg Location, Length and Thickness

\begin{tabular}{c|c|c|c|c|c|c|c}
\hline $\begin{array}{c}\text { Station } \\
\text { Location }\end{array}$ & ID & $\begin{array}{c}\text { Left } \mathbf{X}_{\mathbf{1}} \\
\mathbf{m m}\end{array}$ & $\begin{array}{c}\text { Left } \mathbf{Y}_{\mathbf{1}} \\
\mathbf{M m}\end{array}$ & $\begin{array}{c}\text { Right } \mathbf{X}_{\mathbf{2}} \\
\mathbf{m m}\end{array}$ & $\begin{array}{c}\text { Right } \mathbf{Y}_{\mathbf{2}} \\
\text { mm }\end{array}$ & $\begin{array}{c}\text { Length } \\
\text { mm }\end{array}$ & $\begin{array}{c}\text { Thickness } \\
\text { mm }\end{array}$ \\
\hline Top & Leg 1 & 5700 & 5500 & 7200 & 5500 & 1500 & 250 \\
\hline Bottom & Leg 1 & 5700 & 5500 & 7200 & 5500 & 1500 & 250 \\
\hline
\end{tabular}

Flexural Design for $\mathrm{N}, \mathrm{M}_{2}$ and $\mathrm{M}_{3}$

\begin{tabular}{c|c|c|c|c|c|c|c|c}
\hline $\begin{array}{c}\text { Station } \\
\text { Location }\end{array}$ & $\begin{array}{c}\text { Required } \\
\text { Rebar Area }\left(\mathbf{m m}^{2}\right)\end{array}$ & $\begin{array}{c}\text { Required } \\
\text { Reinf Ratio }\end{array}$ & $\begin{array}{c}\text { Current } \\
\text { Reinf Ratio }\end{array}$ & $\begin{array}{c}\text { Flexural } \\
\text { Combo }\end{array}$ & $\begin{array}{c}\mathbf{N} \\
\mathbf{k N}\end{array}$ & $\begin{array}{c}\mathbf{M}_{\mathbf{2}} \\
\mathbf{k N}-\mathbf{m}\end{array}$ & $\begin{array}{c}\mathbf{M}_{\mathbf{3}} \\
\mathbf{k N}-\mathbf{m}\end{array}$ & $\begin{array}{c}\text { Pier } \mathbf{A}_{\mathbf{g}} \\
\mathbf{m m} \mathbf{2}^{\mathbf{2}}\end{array}$ \\
\hline Top & 938 & 0.0025 & 0.0029 & Comb1 & 914.8321 & -4.5068 & -6.061 & 375000 \\
\hline Bottom & 938 & 0.0025 & 0.0029 & Comb1 & 1029.4589 & -5.124 & -7.7271 & 375000 \\
\hline
\end{tabular}




\begin{tabular}{c|c|c|c|c|c|c|c|c}
\multicolumn{9}{c}{ Shear Design } \\
\hline $\begin{array}{c}\text { Station } \\
\text { Location }\end{array}$ & ID & $\begin{array}{c}\text { Rebar } \\
\mathbf{m m}^{2} / \mathbf{m}\end{array}$ & Shear Combo & $\begin{array}{c}\mathbf{N} \\
\mathbf{k N}\end{array}$ & $\begin{array}{c}\mathbf{M} \\
\mathbf{k N}-\mathbf{m}\end{array}$ & $\begin{array}{c}\mathbf{V} \\
\mathbf{k N}\end{array}$ & $\begin{array}{c}\mathbf{V}_{\mathbf{c}} \\
\mathbf{k N}\end{array}$ & $\begin{array}{c}\mathbf{V}_{\text {total }} \\
\mathbf{k N}\end{array}$ \\
\hline Top & Leg 1 & 319.44 & Comb1.5 & 1372.2482 & 9.0915 & 1.5722 & 272.974 & 392.974 \\
\hline Bottom & Leg 1 & 319.44 & Comb1.5 & 1544.1884 & 11.5906 & 0.0356 & 105.5371 & 225.5371 \\
\hline
\end{tabular}

\section{DISCUSSION:}

In this chapter we will discuss analysis and design results especially effected factors and values obtained like ( response spectrum, diaphragm acceleration, story stiffness, design beams, design columns, design piers ).

\subsection{Response Spectrum Functions:}

response spectrum is a plot of the peak or steady-state response (displacement, velocity or acceleration) of a series of oscillators of varying natural frequency, that are forced into motion by the same base vibration or shock. The resulting plot can then be used to pick off the response of any linear system, given its natural frequency of oscillation. One such use is in assessing the peak response of buildings to earthquakes. The science of strong ground motion may use some values from the ground response spectrum (calculated from recordings of surface ground motion from seismographs) for correlation with seismic damage.

B.S:

Table 2.2 - Response Spectrum Function - UBC 97

\begin{tabular}{|c|c|c|c|c|c|}
\hline Name & $\begin{array}{l}\text { Period } \\
\text { Sec }\end{array}$ & Acceleration & Damping & $\mathbf{C a}$ & $\mathrm{Cv}$ \\
\hline BS RS & 0 & 0.4 & 5 & 0.4 & 0.4 \\
\hline BS RS & 0.08 & 1 & & & \\
\hline BS RS & 0.4 & 1 & & & \\
\hline BS RS & 0.6 & 0.666667 & & & \\
\hline BS RS & 0.8 & 0.5 & & & \\
\hline BS RS & 1 & 0.4 & & & \\
\hline BS RS & 1.2 & 0.333333 & & & \\
\hline BS RS & 1.4 & 0.285714 & & & \\
\hline BS RS & 1.6 & 0.25 & & & \\
\hline BS RS & 1.8 & 0.222222 & & & \\
\hline BS RS & 2 & 0.2 & & & \\
\hline BS RS & 2.5 & 0.16 & & & \\
\hline BS RS & 3 & 0.133333 & & & \\
\hline BS RS & 3.5 & 0.114286 & & & \\
\hline BS RS & 4 & 0.1 & & & \\
\hline BS RS & 4.5 & 0.088889 & & & \\
\hline BS RS & 5 & 0.08 & & & \\
\hline BS RS & 5.5 & 0.072727 & & & \\
\hline BS RS & 6 & 0.066667 & & & \\
\hline BS RS & 6.5 & 0.061538 & & & \\
\hline BS RS & 7 & 0.057143 & & & \\
\hline BS RS & 7.5 & 0.053333 & & & \\
\hline BS RS & 8 & 0.05 & & & \\
\hline BS RS & 8.5 & 0.047059 & & & \\
\hline BS RS & 9 & 0.044444 & & & \\
\hline BS RS & 9.5 & 0.042105 & & & \\
\hline BS RS & 10 & 0.04 & & & \\
\hline
\end{tabular}


I.S:

Table 2.2 - Response Spectrum Function - IS 1893:2002

\begin{tabular}{|c|c|c|c|c|c|}
\hline Name & $\begin{array}{c}\text { Period } \\
\text { sec }\end{array}$ & $\begin{array}{c}\text { Acceleratio } \\
\text { n }\end{array}$ & Damping & Z & Soil Type \\
\hline RS & 0 & 0.24 & 5 & 0.24 & II \\
\hline RS & 0.1 & 0.6 & & & \\
\hline RS & 0.55 & 0.6 & & & \\
\hline RS & 0.8 & 0.408 & & \\
\hline RS & 1 & 0.3264 & & & \\
\hline RS & 1.2 & 0.272 & & \\
\hline RS & 1.4 & 0.233143 & & \\
\hline RS & 1.6 & 0.204 & & \\
\hline RS & 1.8 & 0.181333 & & & \\
\hline RS & 2 & 0.1632 & & \\
\hline RS & 2.5 & 0.13056 & & & \\
\hline RS & 3 & 0.1088 & & \\
\hline RS & 3.5 & 0.093257 & & \\
\hline RS & 4 & 0.0816 & & \\
\hline RS & 4.5 & 0.0816 & & \\
\hline RS & 5 & 0.0816 & & \\
\hline RS & 5.5 & 0.0816 & & \\
\hline RS & 6 & 0.0816 & & \\
\hline RS & 6.5 & 0.0816 & & \\
\hline RS & 7 & 0.0816 & & \\
\hline RS & 8 & 0.0816 & & \\
\hline RS & 8.5 & 0.0816 & & \\
\hline RS & 9 & 0.0816 & & \\
\hline RS & 9.5 & 0.0816 & & \\
\hline RS & 10 & 0.0816 & & \\
\hline & & & & \\
\hline
\end{tabular}

* From above result we saw acceleration values of response spectrum in B.S CODE is higher than I.S CODE.

(B.S CODE approved.)

5.2Diaphragm Accelerations:

accelerations are needed to evaluate in-plane diaphragm forces in earthquake resistant design of buildings, and for the design of their connections. Recorded floor accelerations in buildings during some past earthquakes have shown acceleration magnifications that are not properly considered by current building codes. Earthquake damage in some precast buildings seems to point out significant deficiencies in the design of precast diaphragms.

BS:

\begin{tabular}{|c|c|c|c|c|c|c|c|c|}
\hline Story & Diaphragm & Load Case/Combo & $\begin{array}{c}\mathrm{UX} \\
\mathrm{mm} / \mathrm{sec}^{2}\end{array}$ & $\begin{array}{c}\mathrm{UY} \\
\mathrm{mm} / \mathrm{sec}^{2}\end{array}$ & $\begin{array}{c}\mathrm{UZ} \\
\mathrm{mm} / \mathrm{sec}^{2}\end{array}$ & $\begin{array}{c}\mathbf{R X} \\
\mathrm{rad} / \mathrm{sec}^{2}\end{array}$ & $\begin{array}{c}\mathrm{RY} \\
\mathrm{rad} / \mathrm{sec}^{2}\end{array}$ & $\begin{array}{c}\mathrm{RZ} \\
\mathrm{rad} / \mathrm{sec}^{2}\end{array}$ \\
\hline Story 10 & D10 & RS X Max & 3941.48 & 3992.28 & 1123.85 & 0.622 & 0.383 & 0.185 \\
\hline Story 10 & D10 & RS Y Max & 2433.72 & 2465.09 & 693.93 & 0.384 & 0.237 & 0.114 \\
\hline Story 10 & D10 & TH X Max & 3172.87 & 823.64 & 567.52 & 0.338 & 0.326 & 0.134 \\
\hline Story 10 & D10 & TH X Min & -1909.19 & -779.05 & -585.75 & -0.352 & -0.275 & -0.122 \\
\hline Story 10 & D10 & TH Y Max & 74.87 & 1656.32 & 566.13 & 0.359 & 0.142 & 0.011 \\
\hline Story 10 & D10 & TH Y Min & -74.24 & -1422.08 & -551.6 & -0.276 & -0.158 & -0.011 \\
\hline
\end{tabular}

IS:

Table 3.5 - Diaphragm Accelerations

\begin{tabular}{|c|c|c|c|c|c|c|c|c|}
\hline Story & Diaphragm & $\begin{array}{c}\text { Load } \\
\text { Case/Comb } \\
0\end{array}$ & $\begin{array}{c}\text { UX } \\
\mathrm{mm} / \mathrm{sec}^{2}\end{array}$ & $\begin{array}{c}\mathrm{UY} \\
\mathrm{mm} / \mathrm{sec}^{2}\end{array}$ & $\begin{array}{c}\mathrm{UZ} \\
\mathrm{mm} / \mathrm{sec}^{2}\end{array}$ & $\begin{array}{c}\mathbf{R X} \\
\mathrm{rad} / \mathrm{sec}^{2}\end{array}$ & $\begin{array}{c}\mathrm{RY} \\
\mathrm{rad} / \mathrm{sec}^{2}\end{array}$ & $\begin{array}{c}\mathrm{RZ} \\
\mathrm{rad} / \mathrm{sec}^{2}\end{array}$ \\
\hline Story 10 & D10 & RS X Max & 697.38 & 717.88 & 175.85 & 0.113 & 0.071 & 0.02 \\
\hline Story 10 & D10 & RS Y Max & 701.22 & 721.83 & 176.81 & 0.114 & 0.072 & 0.02 \\
\hline Story10 & D10 & TH X Max & 617.11 & 134.73 & 218.02 & 0.061 & 0.097 & 0.021 \\
\hline Story 10 & D10 & TH X Min & -403.41 & -126.52 & -137.58 & -0.055 & -0.152 & -0.021 \\
\hline Story10 & D10 & TH Y Max & 24.32 & 458.56 & 220.16 & 0.147 & 0.057 & 0.003 \\
\hline Story10 & D10 & TH Y Min & -19.07 & -339.07 & -212.56 & -0.121 & -0.065 & -0.003 \\
\hline
\end{tabular}


*from Above table we took only one story ( story 10 ) we observed that all values of diaphragm acceleration in B.S is higher than values obtained in I.S CODE.

(B.S CODE approved.)

\subsection{Story Stiffness:}

Stiffness is the extent to which an object resists deformation in response to an applied force.

$B S$.

Table 3.10 - Story Stiffness

\begin{tabular}{|l|c|c|c|c|c|c|c|}
\hline Story & Load Case & $\begin{array}{c}\text { Shear X } \\
\mathbf{k N}\end{array}$ & $\begin{array}{c}\text { Drift X } \\
\mathbf{m m}\end{array}$ & $\begin{array}{c}\text { Stiffness X } \\
\mathbf{k N} / \mathbf{m}\end{array}$ & $\begin{array}{c}\text { Shear Y } \\
\mathbf{k N}\end{array}$ & $\begin{array}{c}\text { Drift Y } \\
\mathbf{m m}\end{array}$ & $\begin{array}{c}\text { Stiffness Y } \\
\mathbf{k N} / \mathbf{m}\end{array}$ \\
\hline Story10 & EX 1 & 907.1958 & 8.2 & 110348.034 & 0 & 0.8 & 0 \\
Story10 & EY 1 & 0 & 0.1 & 0 & 562.0547 & 5.6 & 101067.448 \\
Story10 & RS X & 786.3433 & 6.1 & 129834.971 & 825.748 & 6.5 & 126254.367 \\
Story10 & RS Y & 485.5378 & 3.7 & 129834.971 & 509.8688 & 4 & 126254.367
\end{tabular}

IS:

\begin{tabular}{|c|c|c|c|c|c|c|c|}
\hline Story & Load Case & $\begin{array}{c}\text { Shear X } \\
\mathbf{k N}\end{array}$ & $\begin{array}{c}\text { Drift X } \\
\mathbf{m m}\end{array}$ & $\begin{array}{c}\text { Stiffness X } \\
\mathbf{k N} / \mathbf{m}\end{array}$ & $\begin{array}{c}\text { Shear Y } \\
\mathbf{k N}\end{array}$ & $\begin{array}{c}\text { Drift Y } \\
\mathbf{m m}\end{array}$ & $\begin{array}{c}\text { Stiffness Y } \\
\mathbf{k N} / \mathbf{m}\end{array}$ \\
\hline Story10 & EX 1 & 183.7022 & 1.4 & 131506.091 & 0 & 0.03826 & 0 \\
Story10 & EY 1 & 0 & 0.01902 & 0 & 195.153 & 1.5 & 132235.571 \\
Story10 & RS X & 133.8567 & 0.9 & 141454.106 & 143.1019 & 1 & 146446.041 \\
Story10 & RS Y & 134.5933 & 1 & 141454.106 & 143.8894 & 1 & 146446.041 \\
\hline
\end{tabular}

*From above table we observed that shear $\mathrm{X}$ and shear $\mathrm{Y}$ in B.S CODE is higher than shear X and shear Y in I.S CODE for story 10.

*We observed also drift X and Y in B.S CODE is higher than drift X and Y in I.S CODE for story 10.

*We observed also stiffness $\mathrm{X}$ and $\mathrm{Y}$ in B. S CODE is lower than stiffness $\mathrm{X}$ and Y in I.S CODE for story 10.

(I.S CODE approved).

\subsection{Desing of beams:}

A beam is structural elements that primarily resists loads applied laterally to the beam's axis. Its mode of deflection is primarily by bending the loads applied to the beam result in reaction forces at the beam's support points. The total effect of all the forces acting on the beam is to produce shear force and bending moment within the beam, that in turn induce internal stresses, strains and deflections of the beam. Beams are characterized by their manner of support, profile (shape of cross-section), equilibrium conditions, length, and their material.

*From above tables of beam design we observed that required area rebar of I.S code is higher than B.S code then moment values \& required steel bars in I.S code is higher than B.S code.

B.S CODE is approved.

\subsection{Design of columns:}

A column is a vertical structural member intended to transfer a compressive load. For example, a column might transfer loads from a ceiling, floor or roof slab or from a beam to a floor or foundations Columns are typically constructed from materials such as stone brick, block, concrete, timber, steel and so on, which have good compressive strength.

*From above tables of column design we observed that required area rebar of I.S code is higher than B.S code then moment values \& required steel bars in I.S code is higher than B.S code.

B.S CODE is approved.

\subsection{Design of piers:}

In general, it is an upright support for a structure or superstructure, but it can also refer to the sections of loadbearing structural walls between openings and different types of column.Piers are most commonly made of concrete, masonry or treated timber, and installed into prepared holes or shafts.and can also be used in foundations as a means of raising a structure from the ground in particular if the structure is on a slope or near a large body of water.

*From above tables of piers design we observed that required area rebar of I.S code is higher than B.S code then moment values \& required steel bars in I.S code is higher than B.S code.

B.S CODE is approved. 


\section{CONCLUSION:}

- From above tables analysis it is observed that base reaction and center of mass and rigidity values in I.S CODE are higher than values in B.S CODE (T 3.1 \&3.2).

- From above tables analysis it is observed that center of mass displacement and diaphragm acceleration values in B.S CODE are higher than values in I.S CODE ( T $3.3 \& 3.5$ ).

- From above tables analysis it is observed that response spectrum modal information and story max/avg displacement and story drift values in B.S CODE are higher than values in I.S CODE (T3.6 \& $3.7 \& 3.8$ ).

- From above tables analysis it is observed that story forces values in B.S CODE are higher than values in I.S CODE (T3.9).

- From above tables analysis it is observed that story stiffness values in I.S CODE are higher than values in B.S CODE (T3.10).

- From above tables analysis it is observed that modal period and dynamic load participation ratio and modal direction factor values in B.S CODE are higher than values in I.S CODE and frequency and Eigen values in I.S CODE are higher than values in B.S CODE (T3.11\& $3.13 \& 3.14)$.

- From above tables design it is observed that shear force values in I.S CODE are higher than values in B.S CODE until story 7 and for story $8,9 \& 10$ values in B.S CODE are higher than values in I.S CODE.

- From above tables design it is observed that for all stories rebar percentage values in B.S CODE are lower than values in I.S CODE.

- From above tables design it is observed that shear force and reinforcement values in I.S CODE are lower than values in B.S CODE.

images shows:

- Displacement of structure after analysis

- Moment diagram of wind X

- Moment diagram of RSx

- Moment diagram of combo 1.5

- Shear force diagram of combo 1.5

- Response spectrum chart

$$
\mid
$$
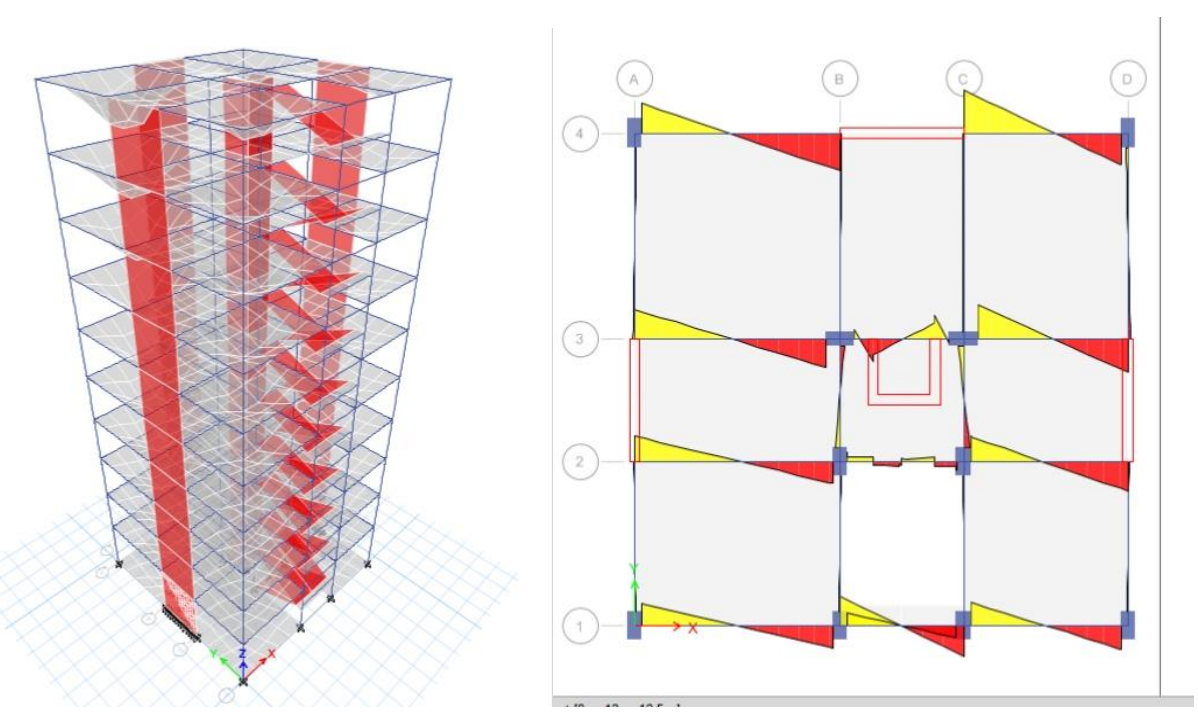

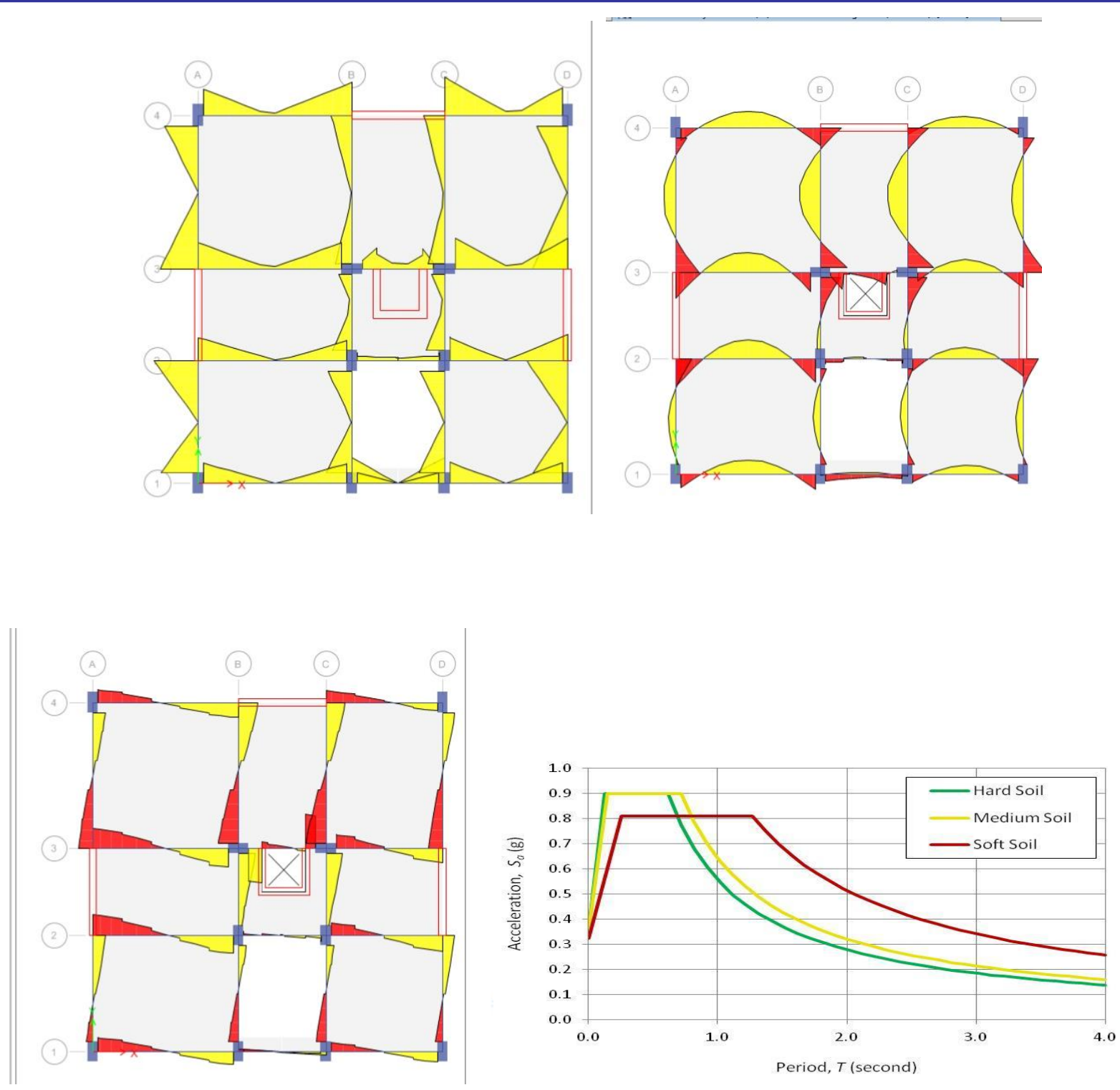

7 REFERENCES:

[1] IS 1893 (part 1): (2002), "Criteria for Earthquake Resistant Design of Structures Part General Provisions and Buildings", Bureau of Indian Standards.

[2] CSI Computers and Structures INC. "Introductory Tutorial for ETABS: Linear and Nonlinear Static and Dynamic Analysis and Design of Three-Dimensional Structures" 2011.

[3] B.C. Punmia, A.K. Jain, 2006, R.C.C Designs”, Laxmi Publications New Delhi.

[4] IS-456 2000 plain and reinforced concrete code of practice.

[5] P.Agarwal, M.Shrinkhande, earthquake resistance design of structures, PHI learning Pvt. 2012.

[6] Pardeshi Sameer, Prof. N. G. Gore (2016), "Study of seismic analysis and design of multi storey symmetrical and asymmetrical building "Volume: 03 Issue: 01

[7] Ali Kadhim Sallal (2018) "Design and analysis ten storied building using ETABS software-2016" Volume 4; Issue 2; May 2018; Page No. 21-27

[8] Pushkar Rathod, Rahul Chandrashekar "seismic analysis of multistoried building for different plans using ETABS 2015" Volume: 04 Issue: $10 \mid$ Oct -2017

[9] S. Vijaya Bhaskar Reddy, Jagath Chandra. P, Srinivas Vasam, P Srinivasa Rao "Analysis Of Multistoried. Structures Using ETABS" Vol. 3, Issue 1, pp: (151-158), Month: April 2015 - September 2015,

[10] B.S 8110 code for RCC. 